\title{
Maternal obesity and gestational diabetes: Impact on arterial wall layer thickness and stiffness in early childhood - RADIEL study six-year follow-up
}

\author{
Johnny K.M. Sundholm ${ }^{\mathrm{a}, *}$, Linda Litwin ${ }^{\mathrm{a}, \mathrm{b}}$, Kristiina Rönö ${ }^{\mathrm{c}}$, Saila B. Koivusalo ${ }^{\mathrm{c}}$, \\ Johan G. Eriksson ${ }^{\text {d,e }}$, Taisto Sarkola ${ }^{a}$ \\ ${ }^{\text {a }}$ Children's Hospital, University of Helsinki, Helsinki University Hospital, Helsinki, Finland \\ ${ }^{\mathrm{b}}$ Department of Congenital Heart Defects and Pediatric Cardiology, SMDZ in Zabrze, SUM, Katowice, Poland

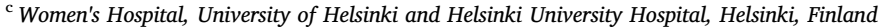 \\ ${ }^{\mathrm{d}}$ University of Helsinki and Helsinki University Hospital, Helsinki, Finland \\ ${ }^{\mathrm{e}}$ Folkhälsan Research Center, Helsinki, Finland
}

\section{H I G H L I G H T S}

- Children to overweight mothers have increased adiposity in early childhood.

- Children to overweight mothers have increased carotid intima-media thickness.

- Impaired glucose balance relates to a minor increase in child carotid stiffness.

- Maternal gestational diabetes (GDM) is not associated with arterial wall layer thickness in childhood.

\section{A R T I C L E I N F O}

\section{Keywords:}

Vascular ultrasound

Pregnancy

Obesity

VHRU

Gestational diabetes

Transgenerational

\begin{abstract}
A B S T R A C T
Background and aims: Gestational diabetes (GDM) and maternal obesity are linked to weight gain in childhood and an increased risk of cardiovascular disease later in life. We assessed the effects of GDM and maternal obesity on arterial function and morphology in relation to body anthropometrics and composition in early childhood. Methods: We assessed body size and composition, blood pressure (BP), arterial morphology and stiffness in 201 pairs of obese mothers (pre-pregnancy BMI $30.7 \pm 5.6 \mathrm{~kg} / \mathrm{m}^{2}, 96$ with GDM) and their children at 6.1 years (SD $0.5)$.

Results: Child BMI (z-score $0.45 \pm 0.92 ; p<0.001$ ) and common carotid intima-media thickness (IMT, z-score $0.15 \pm 0.75, p=0.003$ ) were increased compared with a healthy Finnish reference population. No associations with maternal GDM was found. Carotid IMT and pulse wave velocity were unrelated to child sex, anthropometrics, body composition, BP, as well as maternal anthropometrics and body composition. Carotid stiffness was independently predicted by second trimester fasting glucose. Child lean body mass was the strongest independent predictor for radial (RA), and brachial artery (BA) lumen diameter (LD) and BA IMT (LD: RA: $\mathrm{r}^{2}=0.068, p<0.001$; BA: $\mathrm{r}^{2}=0.108, p<0.001$; IMT: BA: $\left.\mathrm{r}^{2}=0.161, p<0.001\right)$ and carotid LD $\left(r^{2}=0.066, p<0.001\right)$.

Conclusions: Children of obese mothers have increased BMI, blood pressure and carotid IMT suggesting a transgenerational effect of maternal obesity and clustering of cardiovascular risk factors in the population. Arterial dimensions were mainly predicted by child LBM, and not associated with maternal or child adiposity, or GDM. There was a weak association with maternal gestational fasting glucose and increased carotid artery stiffness.
\end{abstract}

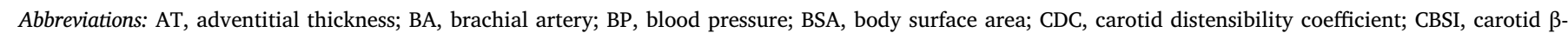

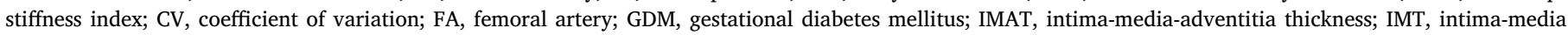
thickness; LBM, lean body mass; PWV, pulse wave velocity; RA, radial artery

* Corresponding author. Children's Hospital, Helsinki University Hospital, Stenbäckinkatu 11, POB 281, FIN-00029, Helsinki, Finland.

E-mail address: johnny.sundholm@helsinki.fi (J.K.M. Sundholm). 


\section{Introduction}

During the last decades, we have seen an increase in obesity and gestational diabetes (GDM) [1-4]. Obesity and GDM impact not only on maternal health but seem to influence the long-term outcome of the child, with early weight gain predicting adult obesity and risk of cardiovascular disease later in life [5-8]. Mechanisms are still partly unknown, but fetal programming has been suggested $[9,10]$.

Carotid artery intima-media thickness (IMT), carotid artery stiffness, and arterial pulse wave velocity (PWV) are independently linked to risk of cardiovascular disease (CVD) $[11,12]$ and are used as surrogate markers to stratify CVD risk in adolescents and adults, but their use in the younger pediatric population is controversial [13-15].

Examination of arterial walls in the youngest pediatric age groups using conventional high-resolution ultrasound is limited due to the minute size of the vascular structures $[16,17]$. Vascular ultrasound using very-high resolution ultrasound frequencies $(25-55 \mathrm{MHz})$ provides a means to quantify carotid and muscular artery wall layer thickness in the youngest children $[17,18]$. The role of muscular artery IMT and adventitia thickness (AT) as markers of CVD is unknown [19], but studies suggest a link between increased radial artery IMT and increased CVD risk [20-22]. To the best of our knowledge, no previous studies have assessed the effect of maternal obesity or GDM on child muscular arteries or muscular artery morphology in relation to CVD risk stratification in early childhood. While multiple studies have covered the subject of child body composition in relation to maternal GDM $[8,10]$, there is only limited data on relations between body composition, BP, blood glucose and lipids, and arterial structure and function in children of GDM and/or obese mothers.

The aim of this study was to assess the transgenerational impact of maternal obesity and GDM on arterial morphology and function in early childhood. We further evaluated associations between child anthropometrics, body composition, BP, blood glucose and lipids with arterial size and function.

\section{Patients and methods}

\subsection{Study setting}

The Finnish Gestational Diabetes Prevention Study (RADIEL) is a randomized controlled multi-center intervention trial set to investigate the effect of a lifestyle intervention for the prevention of GDM. Women in early pregnancy or planning pregnancy with increased risk of GDM due to BMI $\geq 30 \mathrm{~kg} / \mathrm{m}^{2}$ or history of GDM, were prospectively recruited between 2008 and 2012. The subjects were randomized into an intervention group, with intensified structured diet and exercise counselling, or a control group receiving usual care. The study protocol and short term maternal results of the intervention have previously been published $[23,24]$.

This is an observational follow up-study on a subcohort of RADIEL including 201 mother-child pairs (111 boys, 55\%), at an average of 6.1 (0.5 SD) years from delivery (Fig. 1), assessed between June 2015 and May 2017. Maternal and child body anthropometrics, composition, BP, carotid and peripheral artery ultrasound, arterial pulse wave velocity and blood work were assessed. There were no significant effects of RADIEL intervention observed in the subcohort. The local research ethics board and the hospital approved the study. Informed written consent was obtained from the mothers.

\subsection{Maternal gestational characteristics and definition of GDM}

Maternal characteristics were gathered during separate study visits during all trimesters of pregnancy. Information on anthropometrics, socioeconomics and nutrition was prospectively collected and blood work performed during repeat assessments. GDM was diagnosed according to the Finnish guidelines, using a $75 \mathrm{~g} 2 \mathrm{~h}$ oral glucose tolerance test, defined as fasting glucose $>5.3 \mathrm{mmol} / \mathrm{l}, 1 \mathrm{~h}$ glucose $>10.0$ $\mathrm{mmol} / \mathrm{l}$ or $2 \mathrm{~h}$ glucose $>8.6 \mathrm{mmol} / \mathrm{l}$. Dietary treatment was commenced directly at GDM diagnosis, and if morning glucose values exceeded $5.5 \mathrm{mmol} / \mathrm{l}$ or $1 \mathrm{~h}$ postprandial values exceeded $7.8 \mathrm{mmol} / 1 \mathrm{re}$ peatedly, then pharmacological treatment was added $[25,26]$.

\subsection{Vascular ultrasound}

Very-high resolution ultrasound images were obtained by one skilled investigator (TS) using 25, 35 and $55 \mathrm{MHz}$ transducers with the Vevo 770 system, and using UHF22, UHF48 and UHF70 transducers (similar center frequencies) with the Vevo MD system (VisualSonics, Toronto, Canada) for the final 52 mother-child pairs. Images were acquired bilaterally from common carotid and femoral arteries, and from right radial and brachial arteries in the children using $35 / 55 \mathrm{MHz}$ and UHF48/70 transducers. The carotid artery was imaged $1 \mathrm{~cm}$ proximal to the carotid bulb, the radial artery $1 \mathrm{~cm}$ proximal to the palma manus, the brachial artery $3 \mathrm{~cm}$ proximal to the cubital skin fold and the femoral artery at the inguinal fold $1 \mathrm{~cm}$ proximal to the bifurcation into deep and superficial branches. High quality cine images covering 3-4 cardiac cycles were acquired using the highest frequency able to visualize the far-wall. The images were analyzed offline using Vevo 3.0.0 (Vevo 770) and VevoLab (Vevo MD) software with manual electronic calipers [17]. Lumen diameter (LD) and intima media-thickness (IMT) were assessed for all arteries. Intima-media-adventitia thickness (IMAT) was assessed, and adventitia thickness (AT) calculated as the difference between IMAT and IMT, for the muscular radial, brachial and femoral arteries. All dimensions were measured using the leading-edge technique in end-diastole by one experienced observer (JKMS) blinded to subject characteristics. Repeat measurements were independently performed on a subset of images $(\mathrm{N}=10)$ by both primary observer (JKMS) to assess intra-observer variability and by a second observer (TS) to assess inter-observer variability. Intra-observer coefficients of variations (CV) ranged $1.2-2.9 \%$ for LD, 6.9-9.1\% for IMT, 3.4-4.8\% for IMAT and 7.6-12.5\% for AT and inter-observer CV ranged 1.5-4.6\% for LD, $6.0-8.2 \%$ for IMT, $3.5-5.7 \%$ for IMAT and $5.9-12.4 \%$ for AT for the different arteries. Z-scores for vascular dimensions adjusted for age and sex were calculated using reference values for healthy Caucasian non-obese children [27].

Carotid lumen diameter was measured in peak systole and enddiastole to assess carotid artery Beta-stiffness index (CBSI) and carotid artery distensibility coefficient (CDC). Systolic and diastolic BP for elastic property calculations were recorded during ultrasound imaging in supine position from the right arm with oscillometry (Dinamap ProCare 200, GE) using appropriate sized cuffs. CDC and CBSI were calculated from the carotid artery using the formulas:

$C D C=1000 \times \frac{(C C A L A S-C C A L A D) \div C C A L A D}{(B P S-B P D)}$

$C B S I=\frac{\ln \left(\frac{B P S}{B P D}\right)}{(C C A L D S-C C A L D D) / C C A L D D}$

Where CCALAS and CCALAD is common carotid artery lumen area in systole and diastole respectively, CCALDS and CCALDD is common carotid artery lumen diameter in systole and diastole respectively, and BPS and BPD is systolic and diastolic BP [28]. Intra-observer CV was $5.4 \%$ for CDC and 5.9\% for CBSI, and inter-observer CV was $11.9 \%$ for CDC and $12.8 \%$ for CBSI.

\subsection{Pulse wave velocity}

Pulse wave velocity (PWV) was measured by a trained research nurse to assess regional arterial stiffness using mechanosensors (Complior Analyse, Alam Medical, Saint-Quentin-Fallavier, France) at rest in supine position [29]. Sensors were set at the right carotid, right 


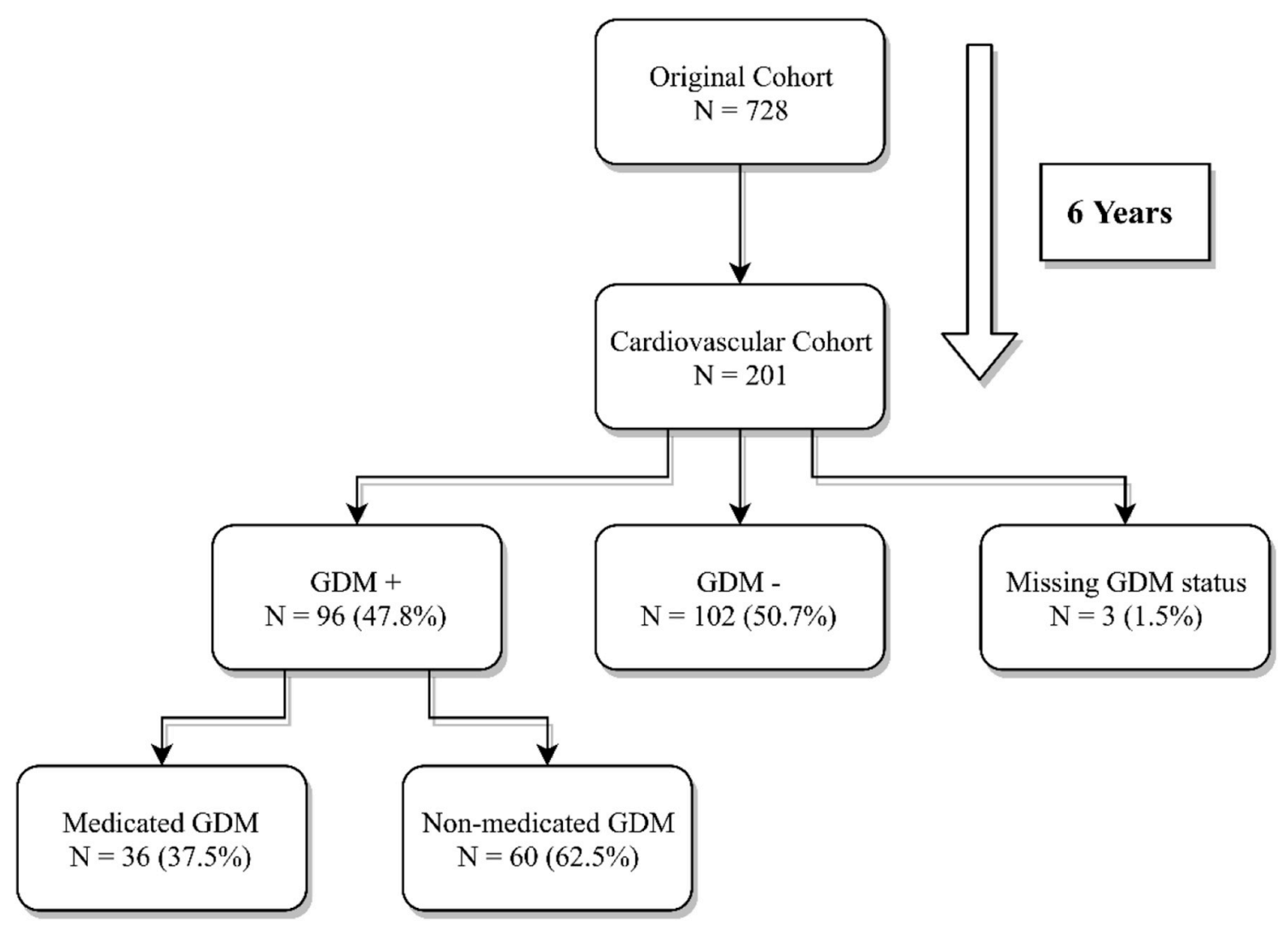

Fig. 1. Flowchart of subject recruitment.

radial and right femoral arteries to assess central (right carotid-femoral) and peripheral (right carotid-radial) transit times. The direct distance between recording sites were measured using a tape measure to the nearest $0.1 \mathrm{~cm}$. The distance was multiplied by 0.8 and subsequently used in PWV calculations. Repeat recordings were performed in supine position. Two recordings were obtained with a third recording performed in the setting of a higher than $0.5 \mathrm{~m} / \mathrm{s}$ (10\%) difference between measurements. In the setting of more than two measurements the results with the lowest tolerance values were used in analyses, tolerance being a quality parameter to quantify variability in pulse waves during recording. The mean of at least two measurements was used in final analyses. PWV was measurable in 168 children. The coefficient of variation (CV) for repeat measurements were $3.5 \%$ for carotid-femoral PWV and $4.8 \%$ for carotid-radial PWV $(\mathrm{N}=55)$.

\subsection{Blood pressure and blood work}

BP was assessed according to NHBPEP 4th report guidelines [30] using three repeat oscillometric measurements (Omron, M6W BP monitor -device, Omron HealthCare Europe, Hoofddorp, Netherlands) at rest in sitting position, using the mean of the two lowest readings. A difference of $<5 \mathrm{mmHg}$ between measures was deemed appropriate. Zscores were generated using the 4 th report reference. [30].

Blood samples were taken by a trained laboratory technician after fasting. Fasting plasma glucose (fP-gluk) was determined using enzymatic hexokinase assay (Roche diagnostics, Basel, Switzerland), Low density lipoprotein (LDL), high density lipoprotein (HDL), triglycerides (TG) and total cholesterol were determined using enzymatic assays, and glycated hemoglobin (HbA1c) was determined using immunoturbidimetric analyzer. The homeostasis model assessment of insulin resistance (HOMA-IR) was calculated (fasting insulin concentration (mU/ 1) $\mathrm{x}$ fasting glucose concentration $(\mathrm{mmol} / \mathrm{l})) / 22.5$ ) [31].

\subsection{Anthropometrics and body composition}

Maternal height and weight was monitored during pregnancy and body composition, in addition, assessed in mothers and children at the 6-year follow up. An electronic stadiometer (Seca gmbh \& co. kg, Hamburg, Germany) was used to measure height to the nearest $0.1 \mathrm{~cm}$. Weight was assessed using an electronic scale (Seca 770, Seca gmbh \& co. $\mathrm{kg}$ ) to the nearest $0.1 \mathrm{~kg}$. Hip and waist circumference were measured with a tape measure to the nearest $0.5 \mathrm{~cm}$. Body surface area (BSA) was calculated using the Mosteller formula [32]. Z-scores for child height and BMI were derived using the recent Finnish growth charts reference population [33]. Overweight and obesity was defined using IOTF criteria [34] and compared to previously published data on Finnish children of a similar age group [35].

Body composition was measured using bioelectrical impedance analysis (Inbody 720, Inbody Bldg., Seoul, South Korea), with a median time difference of 1,0 year (range $-0,05$ to $+2,32$ ) from the cardiovascular assessment. Bioimpedance measurements have previously been shown to be highly correlated with dual energy $\mathrm{x}$-ray absorptiometry in adults, but a systematic bias has been reported, with an underestimation of body fat percentage in children [36,37]. To overcome the issue with methodology and time difference between measurements in growing children a previously validated estimation based on age, sex, height and BMI z-scores at the time of imaging was applied for the children in vascular analyses [38]. To check the agreement between equation predicted and bioimpedance derived lean body mass (LBM) we found a strong correlation between predicted LBM and bioimpedance derived LBM $(r=0,951)$, but with bioimpedance measurements systematically overestimating LBM with a mean difference $2.77 \mathrm{~kg}$ (SD 0.74). After correcting for this bias, the interclass correlation between calculated and measured values was high (ICC $=0.944)$ indicating excellent agreement. Measured bioimpedance derived values were used for mothers. Body fat percentage was calculated as (weight - LBM)/weight. 


\subsection{Data analyses}

Data are presented as mean and standard deviation or as count and percentage. All continuous variables were assessed for normal distributions. GDM positive and negative groups were compared with the independent $t$-test. Subgroups (GDM negative, GDM positive nonmedicated, GDM positive medicated as well as groups stratified by maternal obesity/GDM and child overweight/maternal obesity) were analyzed with the one-way ANOVA and post hoc analysis using Tukey's HSD. Simple univariate correlations were first used to explore associations with vascular parameters.

Multiple linear regression models were used to assess the total effect of maternal pregestational BMI on vascular parameters, adjusted for child sex and age.

Multiple linear regression and ANCOVA models were used to assess total effects of GDM on vascular parameters using different parameters for GDM, i.e. GDM-diagnosis, GDM class (1. GDM negative 2. unmedicated GDM and 3. medicated GDM) and 1st, 2nd, and 3rd trimester fasting glucose, $1 \mathrm{~h}$ and $2 \mathrm{~h}$ OGTT results, and HOMA-IR and adjusted for pregestational BMI as well as either child age and sex or a propensity score included as a covariate in the model. The propensity score was estimated for GDM based on child age, sex, height, weight, BSA, BMI, lean body mass, fat percentage, systolic and diastolic blood pressure. PWV-measurements were further adjusted for heart rate and mean arterial pressure as appropriate [39]. Three mothers with missing information on GDM were excluded from analyses. Statistical analyses were performed with SPSS IBM version 24 and STATA MP 15.1.

\section{Results}

\subsection{Maternal and gestational characteristics}

Maternal characteristics are presented in Supplemental Table 1. The women had a pregestational BMI of $30.7 \pm 5.6 \mathrm{~kg} / \mathrm{m}^{2}(\mathrm{~N}=125 / 201$ with $\geq 30 \mathrm{~kg} / \mathrm{m}^{2}$ ). GDM was diagnosed in $96 / 201$ mothers $(47,8 \%)$, and 36 mothers $(17.9 \%)$ with GDM were medicated orally (metformin) or with insulin during gestation. GDM mothers had higher fasting and OGTT glucose values in both the 1 st and 2 nd trimester. As the spectrum of GDM is broad, ranging from mildly elevated glucose values to its more severe forms, we performed subanalyses on subjects with medicated GDM. The nonmedicated GDM showed only slightly increased glucose values compared to the non-GDM group, while the medicated GDM group had more prominently increased fasting glucose values in the 1st and 2nd trimester (Supplemental Table 1). There was no difference in maternal anthropometrics, body composition or BP in relation to GDM status at follow up. Mothers diagnosed with GDM were more likely to have been diagnosed with GDM in a previous pregnancy and gained less weight during pregnancy (Data not shown).

\subsection{Child characteristics}

Characteristics of the children are presented in Table 1. Children from mothers with medicated GDM had a slightly higher waist-to-hip ratio, but no other significant differences between groups were seen in anthropometrics, body composition or BP. Child BMI and BP were higher than the reference population for all groups. There was a higher incidence of overweight (ISO-BMI 25-30) in both boys (19.8\% vs. $7.3 \%$, $p<0.001)$ and girls $(24.4 \%$ vs. $13.4 \%, p=0.002)$ compared to a reference population. The incidence of obesity (ISO-BMI $>30$ ) was higher among boys $(5.4 \%$ vs. $2.5 \%, p=0.05)$, but not among girls (4.3\% vs. $4.4 \%, p=0.95$ ) compared to a reference population [35]. LBM was higher, fat mass lower and body fat percentage lower in boys compared to girls (all $p<0.001$ ), but no significant sex difference was seen in BMI and BP values (absolute and z-scores).

\subsection{Child vascular parameters}

\subsubsection{Comparison between groups and reference populations}

Results on child arterial dimensions and function stratified for GDM are presented in Supplemental Table 2. There were no statistically significant differences between groups for any parameters. Radial artery LD (z-score: mean 0.16, SD 0.77, $p=0.005$ ) and IMT (z-score: mean $0.17, \mathrm{SD} 1.14, p=0.034$ ) and carotid IMT (z-score: mean 0.16, SD $0.75, p=0.003$ ) were thicker, and carotid LD (z-score: mean -0.33 , SD $0.82, p<0.001$ ) was slightly lower compared to the reference population. There was no statistically significant difference in carotid IMT or carotid artery elasticity when comparing groups of nonobese, obese non-GDM and GDM mothers as well non-overweight, overweight offspring with non-obese and obese mothers, although a weak trend for decreasing elasticity was observed with clustering of maternal obesity, GDM and child overweight (Supplemental Table 3).

\subsubsection{Univariate analysis of vascular parameters}

Univariate associations between maternal gestational adiposity, child sex, age, anthropometrics, body composition, BP, blood glucose and lipids, and arterial dimensions and function were explored with simple correlations (Supplemental Tables 4 and 5). Radial artery LD, brachial artery and femoral artery LD, IMT, and AT correlated with multiple parameters of body size (height, BMI, BSA, waist circumference) and composition (LBM and fat mass). No significant correlation was seen between vascular dimensions and body fat percentage, BP, blood glucose or blood lipids. Radial artery, brachial artery and femoral artery IMT was thicker and femoral artery LD larger in boys compared to girls (all $p<0.001$ ). Only femoral artery AT correlated with maternal pregestational BMI. Carotid LD was correlated with multiple parameters of body size (height, BMI, BSA) and composition (LBM and fat mass) (Supplemental Tables 4 and 5). However, no significant association between sex, body size, composition, BP and carotid IMT was found.

No significant associations between CDC, CBSI, carotid-radial PWV, or carotid-femoral PWV and child sex, body anthropometrics, body composition, blood lipids or glucose was seen (Supplemental Tables 4 and 5). However, CBSI correlated with maternal first and second trimester fasting glucose $(\mathrm{r}=0.221, p=0.003 ; \mathrm{r}=0.250, p=0.002$, Fig. 2). CDC correlated with maternal first trimester insulin resistance index (HOMA-IR: $\mathrm{r}=-0.180, p=0.016$ ), and first and second trimester fasting glucose $(\mathrm{r}=-0.246, p=0.001 ; \mathrm{r}=-0.248$, $p=0.002$, Fig. 2). Similar statistically significant associations were not found between blood glucose and child PWV (Supplemental Table 6).

\subsubsection{Regression and ANCOVA models}

Multiple linear regression and ANCOVA models were used to assess total effects of maternal GDM and maternal pregestational BMI on vascular dimensions and function in the offspring. Maternal pregestational BMI was an independent predictor for femoral AT only (Table 2. Model 1). Multiple linear regression and ANCOVA models including GDM diagnosis, GDM-class, $1 \mathrm{~h}$ and $2 \mathrm{~h}$ OGTT, or HOMA-IR as covariates did not predict any of the vascular parameters. Both CDC and CBSI were predicted by 1 st and 2 nd trimester fasting glucose (Table 2. Model 2-5).

Further linear regression models including child age, sex, LBM, body fat percentage were used to evaluate impact of offspring adiposity on vascular dimensions and function. Significant findings are presented in Table 3. The model was not able to significantly predict carotid dimensions, carotid elasticity, brachial and radial artery AT, or PWV. Child body fat percentage was associated with femoral AT and inversely related to radial IMT, whereas LBM and male sex were positively associated with most vascular dimensions. Age was inversely related to vascular dimensions in models including LBM. This suggests that age 
Table 1

Child characteristics stratified for maternal GDM.

\begin{tabular}{|c|c|c|c|c|c|c|}
\hline \multirow[t]{2}{*}{ Variable } & \multirow[t]{2}{*}{ All mean (SD) or N (\%) } & \multirow[t]{2}{*}{ GDM neg mean (SD) or N (\%) } & \multicolumn{3}{|c|}{ GDM pos mean (SD) or N (\%) } & \multirow[t]{2}{*}{$p$-value GDM pos $v s$. GDM neg } \\
\hline & & & All & Non-medic & Medic & \\
\hline $\mathrm{N}$ & 201 & 102 & 96 & 60 & 36 & \\
\hline Age $[y]$ & $6.09(0.49)$ & $6.04(0.47)$ & $6.11(0.48)$ & $6.21(0.51)$ & $5.95(0.39)$ & 0.30 \\
\hline Male gender & $111(55.2 \%)$ & $58(56.9 \%)$ & $51(53.1 \%)$ & $31(51.7 \%)$ & $20(55.6 \%)$ & 0.67 \\
\hline Height [cm] & $119.1(6.6)$ & $118.9(6.9)$ & $119.3(6.3)$ & $120.3(5.9)$ & $117.7(6.6)$ & 0.65 \\
\hline Height Z-score & $0.11(1.15)$ & $0.11(1.26)$ & $0.12(1.04)$ & $0.20(0.91)$ & $0.02(1.23)$ & 0.99 \\
\hline Weight $[\mathrm{kg}]$ & $23.8(4.1)$ & $23.6(4.3)$ & $23.9(3.9)$ & $23.9(3.9)$ & $23.9(3.9)$ & 0.64 \\
\hline BMI $\left[\mathrm{kg} / \mathrm{m}^{2}\right]$ & $16.7(1.7)$ & $16.6(1.8)$ & $16.7(1.6)$ & $16.4(1.5)$ & $17.2(1.8)$ & 0.64 \\
\hline BMI Z-score & $0.45(0.93)^{\mathrm{b}}$ & $0.41(1.01)$ & $0.5(0.84)$ & $0.35(0.77)$ & $0.76(0.9)$ & 0.5 \\
\hline $\mathrm{BSA}\left[\mathrm{m}^{2}\right]$ & $0.88(0.1)$ & $0.88(0.1)$ & $0.89(0.09)$ & $0.89(0.09)$ & $0.88(0.09)$ & 0.6 \\
\hline Waist-hip ratio & $0.87(0.04)$ & $0.87(0.04)$ & $0.87(0.04)$ & $0.86(0.04)$ & $0.89(0.04)^{\mathrm{a}}$ & 0.85 \\
\hline Waist-height ratio & $0.48(0.03)$ & $0.48(0.03)$ & $0.49(0.03)$ & $0.48(0.03)$ & $0.50(0.04)^{\mathrm{a}}$ & 0.53 \\
\hline Lean body mass [kg] & $16.5(2.4)$ & $16.5(2.5)$ & $16.6(2.3)$ & $16.8(2.3)$ & $16.3(2.2)$ & 0.62 \\
\hline Fat mass $[\mathrm{kg}]$ & $7.2(2.0)$ & $7.2(2.1)$ & $7.3(1.9)$ & $7.1(1.9)$ & $7.6(2.0)$ & 0.71 \\
\hline Body fat percentage [\%] & $30.0(4.0)$ & $30.0(4.1)$ & $30.2(3.8)$ & $29.4(3.5)$ & $31.3(4.2)$ & 0.74 \\
\hline Systolic BP [mmHg] & $99.1(7.0)$ & $98.6(7.5)$ & $99.5(6.5)$ & $99.7(6.5)$ & $99.2(6.6)$ & 0.41 \\
\hline Systolic BP Z-score & $0.41(0.61)^{\mathrm{b}}$ & $0.37(0.66)$ & $0.43(0.57)$ & $0.41(0.55)$ & $0.48(0.6)$ & 0.49 \\
\hline Diastolic BP[mmHg] & $60.7(6.0)$ & $60.9(6.1)$ & $60.6(6.0)$ & $60.6(5.7)$ & $60.7(6.5)$ & 0.78 \\
\hline Diastolic BP Z-score & $0.61(0.56)^{b}$ & $0.62(0.56)$ & $0.61(0.56)$ & $0.56(0.53)$ & $0.68(0.6)$ & 0.86 \\
\hline
\end{tabular}

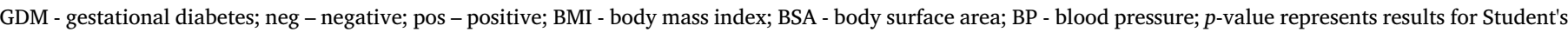
t-test comparison of GDM positive and GDM negative subjects.

a Significance level 0.01 difference between non-medicated and medicated GDM positive subgroups.

b Mean Z-score deviates significantly from population mean.

has a suppressing effect on LBM as a predictor for these vascular parameters, i.e. children with a faster growth during early childhood have increased vascular dimension.

\section{A}

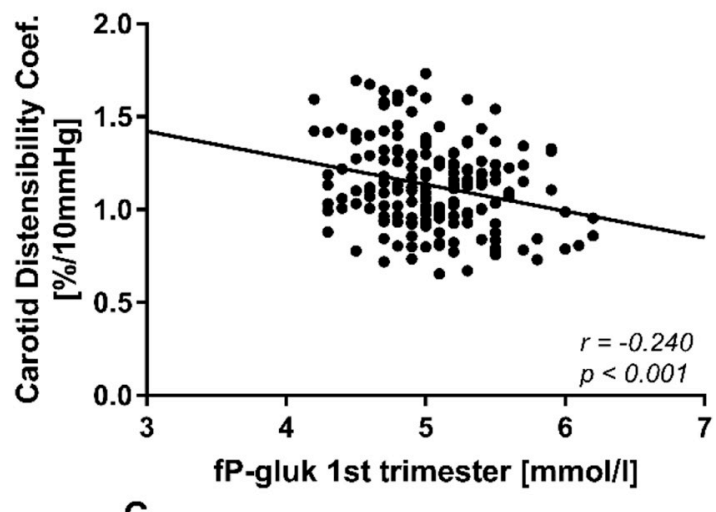

C

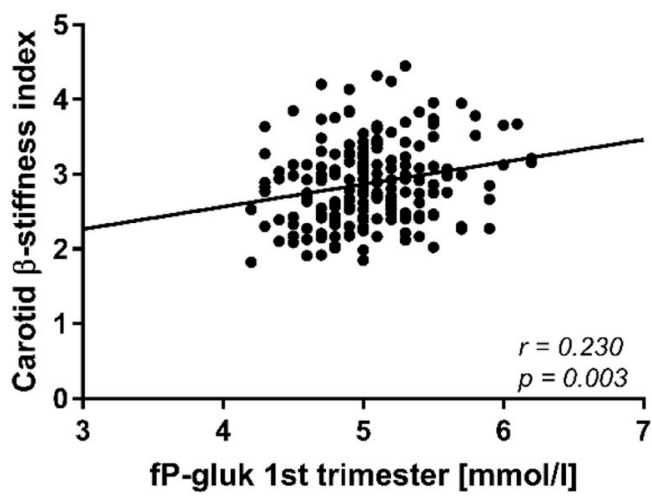

\section{Discussion}

The aim of this study was to investigate the impact of maternal obesity and GDM on offspring vascular morphology and function in
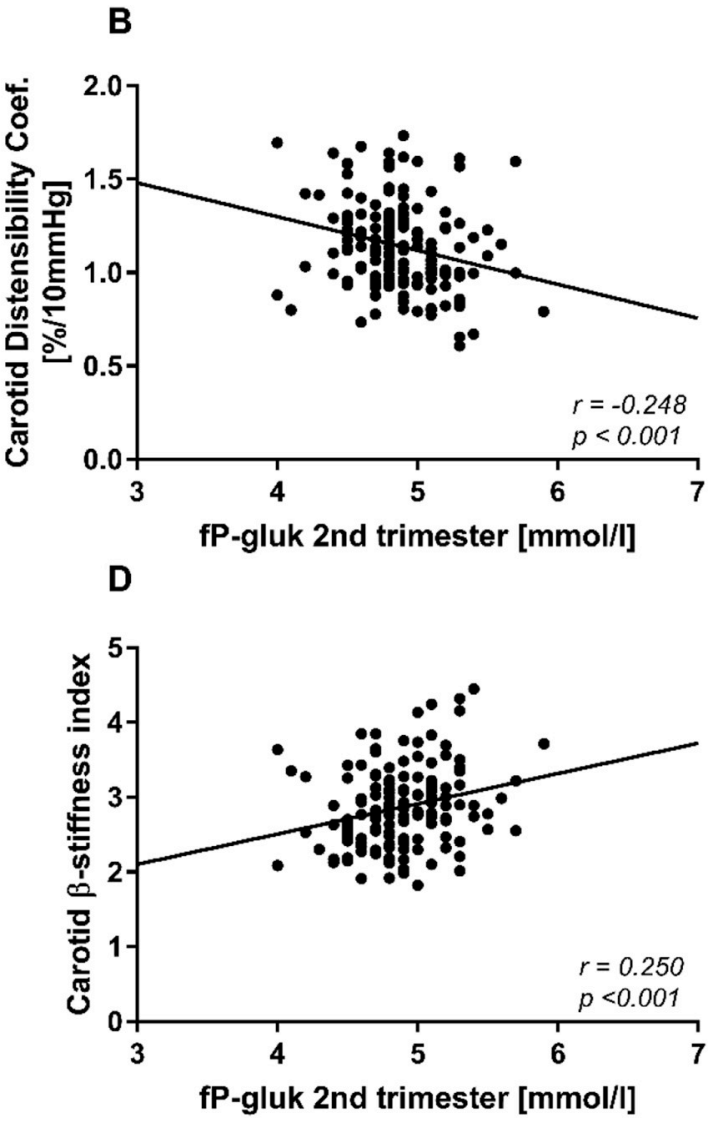

Fig. 2. Scatter plot and linear correlations of child carotid artery stiffness parameters and maternal gestational fasting glucose.

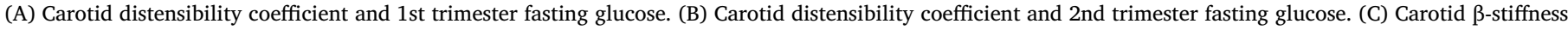
index and 1st trimester fasting glucose. (D) Carotid $\beta$-stiffness index and 2 nd trimester fasting glucose. 
Table 2

Linear regression models total effect of pregestational BMI and GDM on different child vascular parameters.

\begin{tabular}{|c|c|c|c|c|c|}
\hline Model & Variable & Model adjusted R2 & Predictor & Beta & $p$-value \\
\hline \multirow[t]{3}{*}{1} & Femoral adventitia thickness & 0.039 & Pregestational BMI & 1.11 & 0.005 \\
\hline & & & Age & 0.68 & 0.882 \\
\hline & & & Male sex & 5.77 & 0.188 \\
\hline \multirow[t]{4}{*}{2} & Carotid distensibility coefficient $[\% / 10 \mathrm{mmHg}]$ & 0.060 & $1^{\text {st }}$ trimester FPG & -0.139 & 0.001 \\
\hline & & & Age & -0.030 & 0.402 \\
\hline & & & Male sex & 0.009 & 0.795 \\
\hline & & & Pregestational BMI & -0.005 & 0.085 \\
\hline \multirow[t]{4}{*}{3} & Carotid distensibility coefficient $[\% / 10 \mathrm{mmHg}]$ & 0.043 & $2^{\text {nd }}$ trimester FPG & -0.171 & 0.005 \\
\hline & & & Age & 0.009 & 0.811 \\
\hline & & & Male sex & -0.022 & 0.569 \\
\hline & & & Pregestational BMI & -0.004 & 0.293 \\
\hline \multirow[t]{4}{*}{4} & Carotid beta stiffness index & 0.037 & $1^{\text {st }}$ trimester FPG & 0.289 & 0.004 \\
\hline & & & Age & -0.002 & 0.983 \\
\hline & & & Male sex & 0.021 & 0.799 \\
\hline & & & Pregestational BMI & 0.009 & 0.201 \\
\hline \multirow[t]{4}{*}{5} & Carotid beta stiffness index & 0.047 & $2^{\text {nd }}$ trimester FPG & 0.398 & 0.003 \\
\hline & & & Age & -0.085 & 0.335 \\
\hline & & & Male sex & 0.030 & 0.736 \\
\hline & & & Pregestational BMI & 0.008 & 0.331 \\
\hline
\end{tabular}

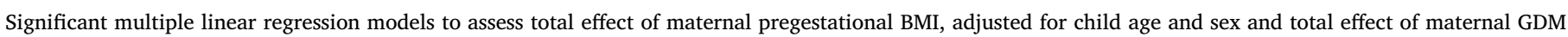
adjusted for child age, sex and maternal pregestational BMI.

early childhood. Additionally, we explored the vascular relations with child anthropometrics, body composition, BP, blood glucose and lipids. We found very limited effects of maternal pregestational BMI (femoral AT only). Most conclusive predictions for arterial dimensions were child lean body mass and male sex, whereas the association with child body fat percentage was limited, suggesting that arterial dimensions are mainly attributed to body growth in line with findings previously shown in infants [40]. A similar relation of vascular dimensions to body size has been shown in early childhood when comparing preterm children with children of normal birth weight [41]. Surprisingly, child carotid IMT was unrelated to child body-size, adiposity or BP in this young homogenous age group. This is partly in line with findings in adolescents were BP, but not adiposity, was shown to be related to carotid IMT [42]. Finally, no effects of the maternal lifestyle intervention to prevent GDM was observed on offspring cardiovascular measures in early childhood.

Nevertheless, there was a weak positive association between local carotid artery stiffness parameters and maternal fasting glucose during the first and second trimester. This association was significant after adjustments for maternal pregestational BMI, and similar trends were found for measurements of insulin-resistance, suggesting an impact of the gestational hyperglycemic milieu on the arterial function. A similar pattern was not, however, seen in regional measurements of arterial stiffness using PWV or in arterial morphology, but these represent a different spectrum of risk for CVD. The effects of GDM on arterial stiffness have previously been studied in adolescence, showing a relationship between hyperinsulinemia and aortic augmentation pressure but not with PWV [43]. It is speculative to say that these findings imply early changes of the vascular tree suggesting increased risk of CVD later in life. Further studies with longer follow-up are needed to verify these findings.

We were unable to show a GDM related difference in child body anthropometrics and body composition, but this early childhood cohort born to mothers with significant obesity had increased BMI, BP, and carotid IMT compared to a healthy reference population. This clustering of CVD risk factors in our cohort of children from obese mothers suggests an increased risk of developing CVD later in life. This is in-line with previous studies in the field showing that maternal obesity is in fact related with adverse CVD outcomes in the child, mainly related to lifestyle and genetics $[44,45]$, whereas the effects of GDM per se have remained controversial $[46,47]$. These results suggest that children born to mothers with significant obesity follow a different growth trajectory, which is reflected in their arterial morphology.

The study is limited by the cohort being recruited as a population with high risk for GDM, with either previous GDM or a BMI $\geq 30$, thus it does not represent the average population, limiting the generalizability of the results to lean populations with GDM. No control population was included, but we matched the reference populations as close as possible to our methods and sample preferring contemporary Finnish population data. Our carotid artery stiffness measurements are limited due to the use of brachial BP in the calculations. However, brachial BP was highly correlated with the estimated carotid BP ( $r$ > 0.95; data available in a subset only), suggesting that brachial BP derived carotid stiffness analyses within our young homogeneous study group reflects true local stiffness. Another limitation is that we do not have any information on the fathers and their potential impact on cardiovascular risk. A clinically relevant difference between GDM groups was challenging to be defined a priori and due to the longitudinal design of the RADIEL study that sample size of this cardiovascular subcohort was estimated to be large enough to enable an extended follow-up beyond 6 years. A post hoc analysis provided a power of 0.8 to detect a difference of 0.5 SD corresponding to a small 6-10\% difference in wall layer thickness or arterial stiffness parameter between groups at a $\alpha$-level of 0.01 . On the other hand, as the groups are highly similar, except with respect to GDM diagnosis, we can draw firm conclusions regarding the lack of an effect of GDM on early vascular morphology in children. The study is strengthened by the prospective design, the large and homogenous sample size and comprehensive assessment of background information, making significant confounding regarding GDM unlikely.

\subsection{Conclusion}

We found no difference in arterial morphology and function between children of obese GDM and non-GDM mothers in early childhood. There was a weak association between maternal gestational fasting glucose and increased carotid artery stiffness in the offspring. Arterial dimensions were mainly related to sex and different parameters of body size, but not with adiposity of the child or mother. The children had a higher BMI, BP and increased carotid IMT compared to a healthy reference population, but independent of the exposure to GDM, suggesting a transgenerational impact of maternal obesity on the child, potentially increasing cardiovascular risk long-term. 
Table 3

Multiple linear regression models assessing effects of child adiposity on vascular dimensions.

\begin{tabular}{|c|c|c|c|c|}
\hline Vascular dimension & Model adjusted R2 & Predictor & Beta & $p$-value \\
\hline \multicolumn{5}{|l|}{ Carotid artery } \\
\hline \multirow[t]{4}{*}{ Lumen diameter $[\mu \mathrm{m}]$} & 0.073 & Age [years] & -70.52 & 0.175 \\
\hline & & Male sex & 98.45 & 0.095 \\
\hline & & Lean body mass $[\mathrm{kg}]$ & 27.26 & 0.051 \\
\hline & & Fat percentage $[\%]$ & 8.56 & 0.181 \\
\hline \multirow[t]{4}{*}{ Intima-media thickness $[\mu \mathrm{m}]$} & 0.008 & Age [years] & 14.18 & 0.059 \\
\hline & & Male sex & 4.31 & 0.548 \\
\hline & & Lean body mass $[\mathrm{kg}]$ & 1.62 & 0.339 \\
\hline & & Fat percentage $[\%]$ & 0.34 & 0.667 \\
\hline \multicolumn{5}{|l|}{ Radial artery } \\
\hline \multirow[t]{4}{*}{ Lumen diameter $[\mu \mathrm{m}]$} & 0.082 & Age [years] & -64.85 & 0.048 \\
\hline & & Male sex & 12.66 & 0.685 \\
\hline & & Lean body mass $[\mathrm{kg}]$ & 21.72 & 0.004 \\
\hline & & Fat percentage $[\%]$ & 2.29 & 0.499 \\
\hline \multirow[t]{4}{*}{ Intima-media thickness $[\mu \mathrm{m}]$} & 0.118 & Age [years] & -1.24 & 0.651 \\
\hline & & Male sex & 4.91 & 0.063 \\
\hline & & Lean body mass $[\mathrm{kg}]$ & 1.35 & 0.029 \\
\hline & & Fat percentage $[\%]$ & -0.71 & 0.014 \\
\hline \multirow[t]{4}{*}{ Adventita thickness $[\mu \mathrm{m}]$} & 0.0069 & Age [years] & 1.19 & 0.664 \\
\hline & & Male sex & -1.92 & 0.463 \\
\hline & & Lean body mass $[\mathrm{kg}]$ & 0.61 & 0.324 \\
\hline & & Fat percentage $[\%]$ & 0.13 & 0.648 \\
\hline \multicolumn{5}{|l|}{ Brachial artery } \\
\hline \multirow[t]{4}{*}{ Lumen diameter $[\mu \mathrm{m}]$} & 0.119 & Age [years] & -60.16 & 0.249 \\
\hline & & Male sex & 63.55 & 0.205 \\
\hline & & Lean body mass $[\mathrm{kg}]$ & 34.81 & 0.004 \\
\hline & & Fat percentage $[\%]$ & 7.00 & 0.196 \\
\hline \multirow[t]{4}{*}{ Intima-media thickness $[\mu \mathrm{m}]$} & 0.183 & Age [years] & 1.23 & 0.648 \\
\hline & & Male sex & 4.10 & 0.114 \\
\hline & & Lean body mass $[\mathrm{kg}]$ & 2.29 & $<0.001$ \\
\hline & & Fat percentage $[\%]$ & -0.23 & 0.418 \\
\hline \multirow[t]{4}{*}{ Adventita thickness $[\mu \mathrm{m}]$} & 0.07 & Age [years] & 3.35 & 0.327 \\
\hline & & Male sex & 1.52 & 0.644 \\
\hline & & Lean body mass $[\mathrm{kg}]$ & 1.25 & 0.109 \\
\hline & & Fat percentage $[\%]$ & 0.46 & 0.192 \\
\hline \multicolumn{5}{|l|}{ Femoral artery } \\
\hline \multirow[t]{4}{*}{ Lumen diameter $[\mu \mathrm{m}]$} & 0.158 & Age [years] & -249.86 & 0.002 \\
\hline & & Male sex & 169.75 & 0.031 \\
\hline & & Lean body mass $[\mathrm{kg}]$ & 60.25 & 0.001 \\
\hline & & Fat percentage $[\%]$ & -0.08 & 0.992 \\
\hline \multirow{4}{*}{ Intima-media thickness $[\mu \mathrm{m}]$} & 0.119 & Age [years] & -11.79 & 0.007 \\
\hline & & Male sex & 8.98 & 0.032 \\
\hline & & Lean body mass $[\mathrm{kg}]$ & 2.86 & 0.004 \\
\hline & & Fat percentage $[\%]$ & 0.12 & 0.799 \\
\hline \multirow[t]{4}{*}{ Adventita thickness $[\mu \mathrm{m}]$} & 0.062 & Age [years] & -4.07 & 0.479 \\
\hline & & Male sex & 9.61 & 0.082 \\
\hline & & Lean body mass $[\mathrm{kg}]$ & 1.66 & 0.216 \\
\hline & & Fat percentage $[\%]$ & 1.20 & 0.047 \\
\hline
\end{tabular}

\section{Conflicts of interest}

The authors declared they do not have anything to disclose regarding conflict of interest with respect to this manuscript.

\section{Financial support}

This study has been supported by grants from the Sigrid Juselius Foundation, Medical Society of Finland, and Foundation for Pediatric Research, Finland, and the Stockmann Foundation.

\section{Author contributions}

Johnny Sundholm assisted in gathering of cardiovascular data during the follow up visit, was responsible for data analysis and management of the follow up data, statistical analysis and writing of the manuscript under supervision of Taisto Sarkola. Linda Litwin assisted with data collection, data management and statistical analysis. Kristiina Rönö managed the data of the primary recruitment and assisted with data management for the follow up study. Saila Koivusalo coordinated and recruited the original cohort. Johan Eriksson coordinated the follow up study and data collection. The cardiovascular data was gathered by Taisto Sarkola, who also supervised data analysis, statistics and writing of the paper. All authors contributed to the writing process and finalization of the manusxript.

\section{Acknowledgements}

The study nurses Maria Finne and Hanna Oksa are acknowledged for the excellent coordination of study visits and data collection management.

\section{Appendix A. Supplementary data}

Supplementary data to this article can be found online at https:// doi.org/10.1016/j.atherosclerosis.2019.01.037.

\section{References}

[1] M Ng, T Fleming, M Robinson, B Thomson, N Graetz, C Margono, et al. Global, regional, and national prevalence of overweight and obesity in children and adults 
during 1980-2013: a systematic analysis for the Global Burden of Disease Study 2013, Lancet. 384 766-781..

[2] Health effects of overweight and obesity in 195 countries over 25 years, N. Engl. J. Med. 377 (2017) 13-27.

[3] C.E. Eades, D.M. Cameron, J.M.M. Evans, Prevalence of gestational diabetes mel litus in Europe: a meta-analysis, Diabetes Res. Clin. Pract. 129 (2017) 173-181.

[4] R. Gaillard, E.A. Steegers, A. Hofman, V.W. Jaddoe, Associations of maternal obesity with blood pressure and the risks of gestational hypertensive disorders. The Generation R Study, J. Hypertens. 29 (2011) 937-944.

[5] M. Akcakus, E. Koklu, A. Baykan, A. Yikilmaz, A. Coskun, T. Gunes, et al., Macrosomic newborns of diabetic mothers are associated with increased aortic intima-media thickness and lipid concentrations, Horm Res Paediatr 67 (2007) $277-283$.

[6] N.A. West, T.L. Crume, Maligie MA, D. Dabelea, Cardiovascular risk factors in children exposed to maternal diabetes in utero, Diabetologia 54 (2011) 504-507.

[7] A. Aceti, S. Santhakumaran, K.M. Logan, L.H. Philipps, E. Prior, C. Gale, et al., The diabetic pregnancy and offspring blood pressure in childhood: a systematic review and meta-analysis, Diabetologia 55 (2012) 3114-3127.

[8] T.D. Clausen, E.R. Mathiesen, T. Hansen, O. Pedersen, D.M. Jensen, J. Lauenborg, et al., Overweight and the metabolic syndrome in adult offspring of women with diet-treated gestational diabetes mellitus or type 1 diabetes, J. Clin. Endocrinol. Metab. 94 (2009) 2464-2470.

[9] P.D. Gluckman, M.A. Hanson, A.S. Beedle, Early life events and their consequences for later disease: a life history and evolutionary perspective, Am. J. Hum. Biol. 19 (2007) 1-19.

[10] R.C. Ma, J.C. Chan, W.H. Tam, M.A. Hanson, P.D. Gluckman, Gestational diabetes, maternal obesity, and the NCD burden, Clin. Obstet. Gynecol. 56 (2013) 633-641.

[11] C. Vlachopoulos, K. Aznaouridis, C. Stefanadis, Prediction of cardiovascular events and all-cause mortality with arterial stiffness: a systematic review and meta-analysis, J. Am. Coll. Cardiol. 55 (2010) 1318-1327.

[12] S. Laurent, J. Cockcroft, L. Van Bortel, P. Boutouyrie, C. Giannattasio, D. Hayoz, et al., Expert consensus document on arterial stiffness: methodological issues and clinical applications, Eur. Heart J. 27 (2006) 2588-2605.

[13] M.H. Park, Á. Skow, S. De Matteis, A.S. Kessel, S. Saxena, R.M. Viner, et al, Adiposity and carotid-intima media thickness in children and adolescents: a systematic review, BMC Pediatr. 15 (2015) 161.

[14] D. White, R. Place, T. Michael, E. Hoffman, P.M. Gordon, P. Visich, The relationship between coronary artery disease risk factors and carotid intima-media thickness in children, J. Pediatr. 190 (2017) 38-42.

[15] M.E. Atabek, H.H. Cagan, B Selver Eklioglu, B. Oran, Absence of increase in carotid artery intima-media thickness in infants of diabetic mothers, J.Clin.Res.Pediatr.Endocrinol. 3 (2011) 144-148.

[16] J.K.M. Sundholm, R.F.W. Olander, T.H. Ojala, S. Andersson, T. Sarkola, Feasibility and precision of transcutaneous very-high resolution ultrasound for quantification of arterial structures in human neonates - comparison with conventional high resolution vascular ultrasound imaging, Atherosclerosis 239 (2015) 523-527.

[17] T. Sarkola, A. Redington, F. Keeley, T. Bradley, E. Jaeggi, Transcutaneous veryhigh-resolution ultrasound to quantify arterial wall layers of muscular and elastic arteries: validation of a method, Atherosclerosis 212 (2010) 516-523.

[18] T. Sarkola, C. Slorach, W. Hui, T.J. Bradley, Redington AN, E. Jaeggi, Transcutaneous very-high resolution ultrasound for the quantification of carotid arterial intima-media thickness in children - feasibility and comparison with conventional high resolution vascular ultrasound imaging, Atherosclerosis 224 (2012) 102-107.

[19] W. Osika, F. Dangardt, J. Grönros, U. Lundstam, A. Myredal, M. Johansson, et al., Increasing peripheral artery intima thickness from childhood to seniority, Arterioscler. Thromb. Vasc. Biol. 27 (2007) 671-676.

[20] A. Vatanen, T. Sarkola, T.H. Ojala, M. Turanlahti, T. Jahnukainen, U.M. SaarinenPihkala, et al., Radiotherapy-related arterial intima thickening and plaque formation in childhood cancer survivors detected with very-high resolution ultrasound during young adulthood, Pediatr. Blood Canc. 62 (2015) 2000-2006.

[21] C. Eklund, E. Omerovic, I. Haraldsson, P. Friberg, L.M. Gan, Radial artery intimamedia thickness predicts major cardiovascular events in patients with suspected coronary artery disease, Eur.Heart J.Cardiovasc.Imaging. 15 (2014) 769-775.

[22] K. Janda, M. Krzanowski, M. Gajda, P. Dumnicka, D. Fedak, G.J. Lis, et al., Cardiovascular risk in chronic kidney disease patients: intima-media thickness predicts the incidence and severity of histologically assessed medial calcification in radial arteries, BMC Nephrol. 16 (2015) 78-7015-0067-8.

[23] K. Rönö, B. Stach-Lempinen, M.M. Klemetti, R.J. Kaaja, M. Pöyhönen-Alho, J.G. Eriksson, et al., Prevention of gestational diabetes through lifestyle intervention: study design and methods of a Finnish randomized controlled multicenter trial (RADIEL), BMC Pregnancy Childbirth 14 (2014) 70-70.

[24] K Rönö SB Koivusalo, M.M. Klemetti, R.P. Roine, J. Lindström, M. Erkkola, et al., Gestational diabetes mellitus can Be prevented by lifestyle intervention: the Finnish gestational diabetes prevention study (RADIEL), Diabetes Care 39 (2016) 24-30.

[25] Gestational Diabetes. Current Care Guidelines. Working Group Set up by the Finnish Medical Society Duodecim and the Finnish Diabetes Association, the Finnish Society of Obstetrics and Gynaecology. Helsinki: The Finnish Medical Society Duodecim, 201 (referred June 6, 2013). Available online at: www.kaypahoito.fi, .

[26] American Diabetes Association, Standards of medical care in diabetes-2008, Diabetes Care 31 (Suppl 1) (2008) S12-S54.

[27] T. Sarkola, C. Manlhiot, C. Slorach, T.J. Bradley, W. Hui, L. Mertens, et al., Evolution of the arterial structure and function from infancy to adolescence is related to anthropometric and blood pressure changes, Arterioscler. Thromb. Vasc. Biol. 32 (2012) 2516.

[28] J.A. Chirinos, Arterial stiffness: basic concepts and measurement techniques, J.Cardiovasc.Transl.Res. 5 (2012) 243-255.

[29] T. Pereira, J. Maldonado, Comparative study of two generations of the Complior device for aortic pulse wave velocity measurements, Blood Press. Monit. 15 (2010) $316-321$.

[30] National high blood pressure education program working group on high blood pressure in children and adolescents. The fourth report on the diagnosis, evaluation, and treatment of high blood pressure in children and adolescents, Pediatrics 114 (2004) 555-576.

[31] D.R. Matthews, J.P. Hosker, A.S. Rudenski, B.A. Naylor, D.F. Treacher, R.C. Turner, Homeostasis model assessment: insulin resistance and beta-cell function from fasting plasma glucose and insulin concentrations in man, Diabetologia 28 (1985) 412-419.

[32] R.D. Mosteller, Simplified calculation of body-surface area, N. Engl. J. Med. 317 (1987) 1098.

[33] A. Saari, U. Sankilampi, M.L. Hannila, V. Kiviniemi, K. Kesseli, L. Dunkel, New Finnish growth references for children and adolescents aged 0 to 20 years: length/ height-for-age, weight-for-length/height, and body mass index-for-age, Ann. Med. 43 (2011) 235-248.

[34] T.J. Cole, T. Lobstein, Extended international (IOTF) body mass index cut-offs for thinness, overweight and obesity, Pediatric Obesity 7 (2012) 284-294.

[35] V. Nina, Saha Marja-Terttu, S. Matti, Prevalence of overweight and obesity in 5- and 12-year-old Finnish children in 1986 and 2006, Acta Paediatr. 98 (2009) 507-512.

[36] L.W. Lee, Y.S. Liao, H.K. Lu, P.L. Hsiao, Y.Y. Chen, C.C. Chi, et al., Validation of two portable bioelectrical impedance analyses for the assessment of body composition in school age children, PLoS One 12 (2017) e0171568, https://doi.org/10.1371/ journal.pone.0171568.

[37] S.L. Faria, O.P. Faria, M.D. Cardeal, M.K. Ito, Validation study of multi-frequency bioelectrical impedance with dual-energy X-ray absorptiometry among obese patients, Obes. Surg. 24 (2014) 1476-1480.

[38] B.J. Foster, R.W. Platt, B.S. Zemel, Development and validation of a predictive equation for lean body mass in children and adolescents, Ann. Hum. Biol. 39 (2012) $171-182$.

[39] R.R. Townsend, I.B. Wilkinson, EL Schiffrin, A.P. Avolio, J.A. Chirinos, J.R. Cockcroft, et al., Recommendations for improving and standardizing vascular research on arterial stiffness: a scientific statement from the American heart association, Hypertension 66 (2015) 698-722.

[40] R.F. Olander, J.K. Sundholm, T.H. Ojala, S. Andersson, T. Sarkola, Neonatal arterial morphology is related to body size in abnormal human fetal growth, Circ.Cardiovasc.Imaging. 9 (2016), https://doi.org/10.1161/CIRCIMAGING.116. 004657.

[41] L.A. Mohlkert, J. Hallberg, O. Broberg, M. Hellstrom, C Pegelow Halvorsen, G. Sjoberg, et al., Preterm arteries in childhood: dimensions, intima-media thickness, and elasticity of the aorta, coronaries, and carotids in 6-y-old children born extremely preterm, Pediatr. Res. 81 (2017) 299-306.

[42] Z. Gao, P.R. Khoury, C.E. McCoy, A.S. Shah, T.R. Kimball, L.M. Dolan, et al., Adiposity has No direct effect on carotid intima-media thickness in adolescents and young adults: use of structural equation modeling to elucidate indirect \& direct pathways, Atherosclerosis 246 (2016) 29-35.

[43] W.H. Tam, R.C. Ma, G.W. Yip, X. Yang, A.M. Li, G.T. Ko, et al., The association between in utero hyperinsulinemia and adolescent arterial stiffness, Diabetes Res. Clin. Pract. 95 (2012) 169-175.

[44] DL Santos Ferreira, D.M. Williams, A.J. Kangas, P. Soininen, M. Ala-Korpela, G.D. Smith, et al., Association of pre-pregnancy body mass index with offspring metabolic profile: analyses of 3 European prospective birth cohorts, PLoS Med. 14 (2017) e1002376.

[45] H.C. Tan, J. Roberts, J. Catov, R. Krishnamurthy, R. Shypailo, F. Bacha, Mother's pre-pregnancy BMI is an important determinant of adverse cardiometabolic risk in childhood, Pediatr. Diabetes 16 (2015) 419-426.

[46] C.J. Nolan, Controversies in gestational diabetes, Best Pract. Res. Clin. Obstet. Gynaecol. 25 (2011) 37-49.

[47] C.B. Williams, K.C. Mackenzie, S. Gahagan, The effect of maternal obesity on the offspring, Clin. Obstet. Gynecol. 57 (2014) 508-515. 